\title{
Validity of mid upper arm circumference for screening acute undernutrition among preschool children in Shimoga, Karnataka
}

\author{
Vijaykumar Mane ${ }^{1, *}$, M.V. Sagar ${ }^{2}$, Revathy $\mathbf{R}^{3}$ \\ ${ }^{\mathbf{1}}$ Assistant Professor, ${ }^{2}$ Professor and Head, ${ }^{3}$ Biostatistician, Dept. of Community Medicine, ${ }^{1}$ Koppal Institute of Medical Sciences, Koppal, \\ Karnataka, ${ }^{2}$ Subbaiah Institute of Medical Sciences, Shimoga, Karnataka, ${ }^{3}$ Shimoga Institute of Medical Sciences, Shimoga, Karnataka, \\ India
}

\section{*Corresponding Author: Vijaykumar Mane}

Email: vijaymane01@ gmail.com

\begin{abstract}
Introduction: Anthropometry is an accepted and most widely used method for assessing the nutritional status of children. Mid Upper Arm Circumference (MUAC) has been recommended to be used as a proxy indicator to screen for acute undernutrition in place of Weight for Height index as it requires no scales, measuring devices or graph plotting.

Objective: To study the validity of mid upper arm circumference in detection of acute undernutrition (wasting) among preschool children in the study setting.

Materials and Methods: A cross sectional study was conducted in the field practice area of PHC Aaynoor, Shimoga District, Karnataka state for a duration of 1 year from January 2013 to December 2013. Cluster sampling technique was used for sample selection and 400 preschool children of either sex in the age group of 1-5 years (12- 59 months) were included in the study. Anthropometric measurements were recorded using standard techniques.

Results: The prevalence of both moderate and severe wasting as detected by MUAC was $27(6.75 \%)$ and $3(0.75 \%)$, which is less than half of that detected by WHZ. MUAC had a sensitivity of $23.2 \%$ and $14.3 \%$ respectively in detection of moderate and severe wasting. It could identify higher number of wasted children among males and among children aged $12-23$ months.

Conclusion: Current MUAC cut offs are inappropriate for detection of wasting among preschool children.
\end{abstract}

Keywords: Malnutrition, Mid upper arm circumference, Validity, Sensitivity \& specificity.

\section{Introduction}

Good nutrition is prerequisite for child survival, health and development. Adequate nutrition allows children to grow, develop, learn, play, participate and contribute to their communities and to be resilient in the face of disease, disasters and other crisis. Further, economic growth and human development require well-nourished populations who can learn new skills, think critically and contribute to their communities. ${ }^{1,2}$

The level of child undernutrition remains unacceptably high throughout the world. Undernutrition puts children at greater risk of dying from common infections, increases the frequency and severity of such infections, and contributes to delayed recovery. Nearly half of all deaths in children under 5 are attributable to undernutrition. Undernutrition also impacts cognitive function and contributes to poverty through impeding individual's ability to lead productive lives. ${ }^{2,3}$

Anthropometry is an accepted and most widely used method for assessing the nutritional status of children. The gold standard to identify acute malnutrition is weight-forheight z-score (WHZ) below -2 standard deviations of the international reference population (World Health Organization 2006 Growth Standards). Unfortunately, scales and/or height board are not always available for screening at community level in many developing countries. As subcutaneous fat and muscle mass decrease in proportion to loss of body weight, Mid Upper Arm Circumference (MUAC) has been recommended to be used as a proxy indicator to screen for acute malnutrition. ${ }^{4-8}$
MUAC is a valuable form of low cost technology applicable at village health worker level as it requires no scales, measuring devices or graph plotting. The MUAC cutoff points were selected from statistical analysis of nutritional surveys to approximately correspond to the WHZ cut-off points so that the same prevalence of acute malnutrition would be found and should identify the same undernourished children. However, in practice, there is a discrepancy between the prevalence of children identified as malnourished by WHZ and by MUAC. ${ }^{4,8}$ Since there have been reports indicating that the proportion of children identified by both criteria varies from country to country and as there is paucity of data in Indian settings, ${ }^{8,9}$ the following study was undertaken.

\section{Objective of the Study}

To study the validity of mid upper arm circumference in detection of acute undernutrition (wasting) among preschool children in the study setting.

\section{Materials and Methods}

A cross sectional study was conducted in the field practice area of PHC Aaynoor, Shimoga District, Karnataka state for a duration of 1 year from January 2013 to December 2013. Cluster sampling technique was used for sample selection. All the 30 villages coming under PHC Aaynoor were considered as clusters and the same constituted sampling units for the study. 20 clusters were selected by population proportional to size sampling. In each cluster, house to house visit was done and 20 preschool children were included, thus making a total sample of 400. Temporary 
visitors/ guests to the house and children unavailable in the house at the time of visit were excluded from the study. Assent of the child and consent of their parents was taken before taking anthropometric measurements and data was collected in a pretested and semi-structured questionnaire.

\section{Anthropometric Measurements}

Weight: The weight of the child was taken using a LED digital portable weighing scale (SAMSO) to the nearest 0.1 $\mathrm{kg}$ for accuracy. The study subjects were weighed with minimal clothing and without footwear. Children who were unable to stand still on weighing scale, were weighed together with the mother and the weight of the mother was subtracted to obtain the weight of the child.

Height/Length: A non-flexible metallic tape was used to measure length for children aged less than two years and height for those aged 2-5 years. Measurements were taken to the nearest centimeters for accuracy. Child was made to lie on a firm flat surface, head was positioned such that the eyes are looking vertically upwards (i.e. Frankfurt plane positioned vertically), knees extended by applying firm pressure and feet are flexed at right angles to the legs and length was recorded. For measurement of height, child was made to stand on flat floor looking straight ahead with bare feet placed slightly apart and the back of the head, shoulder blades, buttocks, calves and heels touching the upright wall. The arms were made to hang at sides in natural manner and the reading was taken.

Mid Upper Arm Circumference: Mid upper arm circumference (MUAC) was measured using flexible measuring tape. The midpoint was assessed by measuring the distance between the acromial process of scapula and the olecranon process of ulna and taking the midpoint of that distance. Then the circumference of the left arm is measured at this point using the flexible measuring tape to the nearest 0.1 centimeter.

\section{Statistical Analysis}

Detection of acute undernutrition (wasting) was done using WHO Anthro-software version 3.2.2. It compares the anthropometric measurements of each child with the WHO child growth standards, 2006 data for that particular age and sex to get weight for height index. Children with WHZ index below two standard deviation of the reference median were considered as undernourished and termed as wasted and those below three standard deviation were considered as severely undernourished and termed severely wasted. ${ }^{10}$

Mid upper arm circumference exceeding $125 \mathrm{~mm}$ was considered as satisfactory nutritional status and values below the cut-offs of $125 \mathrm{~mm}$ and $115 \mathrm{~mm}$ were used to define wasting and severe wasting as per WHO criteria. ${ }^{11}$ Data thus obtained was entered and analyzed using WHO Epi-Info software version 3.5.4.

\section{Results}

Fig. 1: shows prevalence of acute malnutrition (wasting) with both indices - Weight for Height index and mid upper arm circumference. It is evident from the figure that the prevalence of both moderate and severe wasting as detected by MUAC was $27(6.75 \%)$ and $3(0.75 \%)$, which is less than half of that detected by WHZ i.e. $56(14 \%)$ and $7(1.75 \%)$ respectively.

Table 1 (a) and (b): demonstrates the validity of MUAC in detection of moderate and severe wasting against WHZ respectively. Out of 56 moderately wasted children, MUAC could detect only 13 , which gives a sensitivity of $23.2 \%$ and similarly out of 7 severely wasted children, MUAC could identify only 1 , thereby showing a sensitivity of $14.3 \%$. However, MUAC had a very good specificity of above $95 \%$ in detection of both the degrees of wasting.

Table 2: demonstrates validity of MUAC in detection of wasting against WHZ according to gender. It is evident from the table that MUAC could detect only 11 of 31 wasted children among males and 7 of 32 among females, thereby showing a sensitivity of $35.5 \%$ and $21.9 \%$ respectively.

Table 3: shows validity of MUAC in detection of wasting against WHZ in different age groups. MUAC had highest sensitivity of detecting $50 \%$ of wasting among children aged 12 - 23 months followed by a sensitivity of $33.3 \%$ and $20 \%$ among children aged $36-47$ months and $24-35$ months respectively as seen in the table.

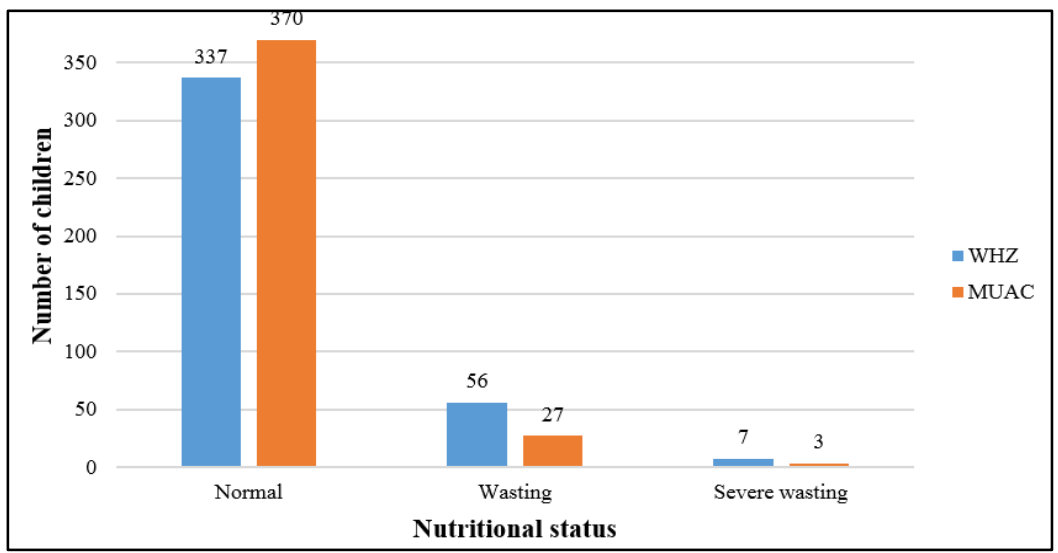

Fig. 1: Nutritional status of study participants using 2 indices - Weight for height $\mathrm{z}$ score (WHZ) and Mid upper arm circumference (MUAC) 
Table 1 (a): Validity of MUAC in detection of moderate wasting against WHZ

\begin{tabular}{|c|c|c|c|c|}
\hline Nutritional & idices & & & \\
\hline & & Positive & Negative & Total \\
\hline $12.5>$ MUAC $\geq 11.5$ & Positive & $13(23.2)$ & $14(4.1)$ & 27 \\
\hline & Negative & $43(76.8)$ & $330(95.9)$ & 373 \\
\hline & Total & $56(100)$ & $344(100)$ & 400 \\
\hline
\end{tabular}

Table 1 (b): Validity of MUAC in detection of severe wasting against WHZ

\begin{tabular}{|c|c|c|c|c|}
\hline \multicolumn{2}{|c|}{ Nutritional status indices } & \multicolumn{2}{|c|}{ WHZ <-3 } & Tegative \\
\cline { 3 - 5 } & Positive & $2(0.5)$ & 3 \\
\hline \multirow{2}{*}{ MUAC $<\mathbf{1 1 . 5}$} & Positive & $1(14.3)$ & $391(99.5)$ & 397 \\
\cline { 2 - 5 } & Negative & $6(85.7)$ & $393(100)$ & 400 \\
\hline
\end{tabular}

Table 2: Validity of MUAC in detection of wasting against WHZ (Gold standard) according to gender

\begin{tabular}{|c|c|c|c|c|c|}
\hline \multirow{3}{*}{\multicolumn{2}{|c|}{ Nutritional status }} & \multicolumn{4}{|c|}{ Weight for Height $\mathrm{Z}$ score $\mathrm{N}(\%)$} \\
\hline & & \multicolumn{2}{|c|}{ Male $(n=190)$} & \multicolumn{2}{|c|}{ Female $(\mathbf{n}=210)$} \\
\hline & & Normal & Wasting & Normal & Wasting \\
\hline \multirow{3}{*}{$\begin{array}{l}\text { Mid upper arm } \\
\text { circumference }\end{array}$} & Normal & $156(98.1)$ & $20(64.5)$ & $169(94.9)$ & $25(78.1)$ \\
\hline & Wasting & $3(1.9)$ & $11(35.5)$ & $9(5.1)$ & $7(21.9)$ \\
\hline & Total & $159(100)$ & $31(100)$ & $178(100)$ & $32(100)$ \\
\hline
\end{tabular}

Table 3: Validity of MUAC in detection of wasting against WHZ (Gold standard) in different age groups

\begin{tabular}{|c|c|c|c|c|c|c|c|c|c|}
\hline \multirow{3}{*}{\multicolumn{2}{|c|}{ Nutritional status }} & \multicolumn{8}{|c|}{ Weight for Height $\mathrm{Z}$ score $\mathrm{N}(\%)$} \\
\hline & & \multicolumn{2}{|c|}{$\begin{array}{c}12-23 \text { months } \\
(n=112)\end{array}$} & \multicolumn{2}{|c|}{$\begin{array}{c}24-35 \text { months } \\
(n=101)\end{array}$} & \multicolumn{2}{|c|}{$\begin{array}{c}36-47 \text { months } \\
(n=97)\end{array}$} & \multicolumn{2}{|c|}{$\begin{array}{c}48-59 \text { months } \\
(n=90)\end{array}$} \\
\hline & & Normal & Wasting & Normal & Wasting & Normal & Wasting & Normal & Wasting \\
\hline \multirow{3}{*}{ MUAC } & Normal & $81(90)$ & $11(50)$ & $89(97.8)$ & $8(80)$ & $84(98.8)$ & $8(66.7)$ & $71(100)$ & $18(94.7)$ \\
\hline & Wasting & $9(10)$ & $11(50)$ & $2(2.2)$ & $2(20)$ & $1(1.2)$ & $4(33.3)$ & $0(0)$ & $1(5.3)$ \\
\hline & Total & $90(100)$ & $22(100)$ & $91(100)$ & $10(100)$ & $85(100)$ & $12(100)$ & $71(100)$ & $19(100)$ \\
\hline
\end{tabular}

\section{Discussion}

Mid-upper arm-circumference has been considered as a simple, quick and easier screening tool at community level for detection of wasting among children aged 1 to 5 years of age and the present study is an attempt to test the accuracy of MUAC against Weight for Height in detection of wasting keeping in mind a number of studies reporting discrepancies between the two indicators. The prevalence of both moderate and severe wasting as detected by MUAC cut off values was less than half of that detected by WHZ score in the present study and is consistent with the findings of many other studies. . $^{6,7,9,12-16}$

The sensitivity of MUAC cut off value in detecting moderate wasting was $23.2 \%$ against WHZ score similar to the findings of many other studies ${ }^{6,7,13-15,17}$ and was $14.3 \%$ in detection of severe wasting as reported by few other studies. ${ }^{6,7,9,13-15}$ On the other hand MUAC had a specificity of more than $95 \%$ in detection of both moderate and severe wasting, which confirms the findings of other studies. ${ }^{6,9,13-}$ 15,17

MUAC had a little higher sensitivity among male children in the present study contrary to the findings of studies done in Cambodia ${ }^{7}$ and Nigeria, ${ }^{14}$ which could be due to differences in geographic location of study settings. Sensitivity of MUAC was also found to be highest in children aged 12-23 months in the present study similar to the findings of studies done in other countries. ${ }^{7,12,18}$

\section{Conclusion}

The present study findings conclude that MUAC though simple does not appear to be appropriate tool for the diagnosis of wasting among preschool children and stresses the need to introspect on the suitability of current MUAC cutoffs.

\section{Acknowledgement}

Authors acknowledge faculty from the department of Community Medicine, Shimoga Institute of Medical Sciences, Shimoga, Karnataka for overall motivation and support. Authors also thank Administrative Medical Officer, PHC Aaynoor and all the field workers for their help and cooperation in conducting the study.

\section{Conflict of Interest: Nil.}

\section{References}

1. "Nutrition." Available at URL

https://www.unicef.org/nutrition/ Accessed on 06/08/2017

2. United Nations Children's Fund, World Health Organization, The World Bank. UNICEF WHO- World Bank joint child malnutrition estimates. UNICEF, New York; WHO, Geneva; The World Bank, Washington, DC;2012;9-10. 
3. "Undernutrition contributes to nearly half of all deaths in children under 5 and widespread in Asia and Africa." Available at URL https://data.unicef.org/topic/nutrition/malnutrition/ Accessed on $06 / 08 / 2017$

4. Kumar R, Aggarwal AK, Iyengar SD. Nutritional status of children: Validity of Mid Upper arm circumference for screening undernutrition. Indian Pediatr. 1996;33:189-196.

5. "Measuring Malnutrition" Available at URL https://www.unicef.org/nutrition/training/3.1/5.html_Accessed on $06 / 08 / 2017$

6. Mane VP, Naik TB, Mallappa O, Ambure O. Validity of Mid Upper arm circumference for screening undernutrition among preschool children. Indian J Forensic Community Med. 2015;2(3):144-146.

7. Fiorentino M, Sophonneary P, Laillou A, Whitney S, de Groot $\mathrm{R}$, Perignon M, et al. Current MUAC Cut-Offs to Screen for Acute Malnutrition Need to Be Adapted to Gender and Age: The Example of Cambodia. PLoS ONE. 2016;11(2): e0146442.doi:10.1371/journal.pone.0146442

8. Grellety E, Golden MH. Weight - for - Height and Mid Upper - arm - circumference should be used independently to diagnose acute malnutrition: Policy implications. BMC Nutrition 2016;2(10): DOI 10.1186/s40795-016-0049-7.

9. Tripathy JP, Sharma A, Prinja S. Is Mid upper arm circumference alone sufficient to Identify Severe Acute Malnutrition correctly? Indian Pediatr. 2016;53:166-167.

10. World Health organization, Department of Nutrition for Health and Development. WHO child growth standards: Training course on Child growth assessment. World health organization 2008. Module - C:22.

11. World Health Organization, United Nations Children's Fund. WHO child growth standards and the identification of severe acute malnutrition in infants and children $\mathrm{A}$ joint statement by the World Health Organization and the United Nations Children's Fund. WHO 2009.

12. Bern C, Nathanail L. Is mid upper arm circumference a useful tool for screening in emergency settings? Lancet. 1995:345;631-633.
13. Dasgupta R, Sinha D, Jain SK, Prasad V. Screening for SAM in the community: Is MUAC a 'Simple Tool'? Indian Pediatr. 2013;50:154-155.

14. Dairo MD, Fatokun ME, Kuti M. Reliability of the Mid Upper Arm Circumference for the Assessment of Wasting among Children Aged 12-59 Months in Urban Ibadan, Nigeria. Int J Biomed Sci. 2012:8(2);140-143.

15. Laillou A, Prak S, de Groot R, Whitney S, Conkle J. Optimal Screening of Children with Acute Malnutrition Requires a Change in Current WHO Guidelines as MUAC and WHZ Identify Different Patient Groups. PLoS ONE. 2014;9(7): e101159. doi:10.1371/journal.pone.0101159

16. Natisha Dukhi, Benn Sartorius \& Myra Taylor (2017) Midupper arm circumference (MUAC) performance versus weight for height in South African children (0-59 months) with acute malnutrition. S Af J Clin Nutr, 2017;30(2):49-54. DOI:10.1080/16070658.2016.1255483

17. Brahmbhatt KR, Hameed S, Naik PM, Prasanna KS, Jayram S. Role of new anthropometric indices, validity of MUAC and Weech's formula in detecting under-nutrition among underfive children in Karnataka. Int J Biomed \& Adv Res. 2012:3(12);896-900.

18. Hop LT, Gross R, Sastroamidjojo S, Giay T, Schultink W. Mid-upper arm circumference development and its validity in assessment of Undernutrition. Asia Pacific J Clin Nutr. 1998:7(1);65-69.

How to cite this article: Mane V, Sagar M.V, Revathy R. Validity of mid upper arm circumference for screening acute undernutrition among preschool children in Shimoga, Karnataka. Indian J Forensic Community Med. 2018;5(4):227-230. 